\title{
A three-step pattern in audiometric thresholds
}

\author{
Eric C. Hoover ${ }^{1}$ \\ Department of Hearing and Speech Sciences, University of Maryland, 7251 Preinkert Drive, \\ College Park, Maryland 20742, USA \\ ehoover@umd.edu
}

\begin{abstract}
Use of the audiogram as the gold standard index of hearing ability amplifies the consequences of error in threshold measurement. A Markov chain model of the audiometric procedure revealed a three-step pattern in the stimuli presented each trial. Monte Carlo simulations were used to generate threshold estimates for a simple listener model. Thresholds sorted by trial had a mean bias consistent with model predictions. An alternate scoring method is proposed that uses equal sampling of Markov states. The resulting threshold targets a specific probability of detection and has no systematic bias as a function of trial.
\end{abstract}

\section{Introduction}

Improving the measurement of the limit of audibility has been a topic of continuous interest throughout the history of hearing research (Bunch, 1943; Hirsh, 1952). Since the standardization of audiometry, there have been notable breakthroughs in our ability to accurately calibrate and reliably present a specific sound pressure in the ear (e.g., Flamme, et al. 2015; Green, et al. 1987; Souza, et al. 2014). However, limitations in the ability of the audiometric procedure to accurately characterize listener behavior remains the dominant contributor to error in threshold estimates (Green, et al. 1987; Marshall, et al. 1996; Meddis \& Lecluyse, 2011; Stelmachowicz et al., 1988; 1989). In this report a novel source of procedural error is described. The error arises because of a three-step pattern in the stimulus levels that can be presented each trial. Fortunately, the error occurs only when a single stimulus level presented during a track is reported as threshold. The

\footnotetext{
${ }^{1}$ Author to whom correspondence should be addressed.
} 
error can be eliminated by computing threshold from the average of multiple stimulus levels presented during a track as is typically done in laboratory psychoacoustics.

The procedure used to obtain audiometric thresholds is as follows (Carhart \& Jerger, 1964; ANSI, 2004; ISO, 2010): present a tone that is clearly audible to the listener, decrease the level $10 \mathrm{~dB}$ after a hit (correct response) and increase it $5 \mathrm{~dB}$ after a miss (no response), and stop after at least two hits and hits in half of trials at a stimulus level presented in ascending runs. The lowest level that met the stopping criteria is reported as threshold. The method is highly efficient compared to laboratory procedures (Marshall et al., 1996; Lecluyse \& Meddis, 2009); thresholds can be obtained in as few as five and rarely more than fifteen trials (Harris, 1979; Jerlvall \& Arlinger, 1986; Marshall \& Hanna 1986). With so few trials, high efficiency can be achieved despite the large error in repeated estimates. However, there are critical applications for which the magnitude of the error is a limiting factor, including detecting a change in hearing (e.g., ototoxicity monitoring), and controlling audibility (e.g., studying the effects of aging on speech perception). Identification and elimination of sources of error in threshold estimates have potentially profound consequences for clinical and research applications of the audiogram.

A meta-analysis of studies reporting test-retest variability in repeated audiograms found an error of $3.4 \mathrm{~dB}$ at $1000 \mathrm{~Hz}$ (Mahomed et al., 2013). This means that, on average, repeated audiograms performed in the same individual with no change in hearing reflect a Gaussian distribution with a SD of $2.4 \mathrm{~dB}$. However, a threshold reported on an audiogram is not typically the mean of repeated tests, it is the result of a single test, and it represents a single stimulus level presented during that test. An audiogram displays only the stimulus levels that can be presented during a track (e.g., $5 \mathrm{~dB}$ HL, not $3.14 \mathrm{~dB}$ HL). While the $5 \mathrm{~dB}$ difference between possible stimulus levels can be deceased by decreasing the step size, as recommended by Leijon (1992), decreasing step size does not decrease the SD of repeated thresholds or the test-retest error (Jerlvall \& Arlinger, 1986; Leijon, 1992; Marshall et al., 1996). In fact, the difference between stimulus levels that can be presented on a given trial is $15 \mathrm{~dB}$, not $5 \mathrm{~dB}$. It is demonstrated in the following section that only one third of possible stimulus levels can be presented on each trial. 


\subsection{Three-step pattern}

A pattern can be observed in the sequence of stimulus levels presented in a typical audiometric test. Figure 1, left panel, shows an example sequence starting at $30 \mathrm{~dB} \mathrm{HL}$ and resulting in a threshold of $15 \mathrm{~dB} \mathrm{HL}$. Each stimulus level presented during the test was sorted into three columns in Figure 1, middle panel. A pattern can be observed in the stimulus levels in each column. Stimuli in column A are multiples of $15 \mathrm{~dB}$ and do not appear in any other column. Likewise, stimuli in column B are separated by $15 \mathrm{~dB}$, and no stimuli appear in more than one column. Regardless of whether the response on any trial was a hit or a miss, the next stimulus presented always followed the pattern for stimuli in the next column.

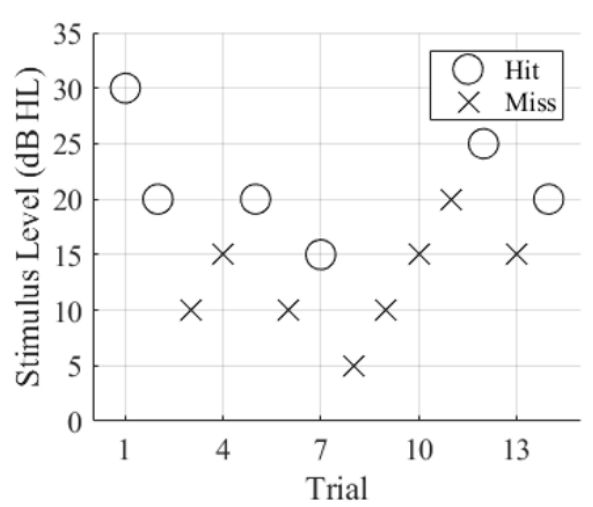

\begin{tabular}{|c|c|c|}
\hline $\mathrm{A}$ & B & $\mathrm{C}$ \\
\hline Trial 1 & Trial 2 & Trial 3 \\
\hline $30 \mathrm{~dB} \mathrm{HL}$ & $20 \mathrm{~dB} \mathrm{HL}$ & $10 \mathrm{~dB} \mathrm{HL}$ \\
\hline Trial 4 & Trial 5 & Trial 6 \\
\hline $15 \mathrm{~dB} \mathrm{HL}$ & $20 \mathrm{~dB} \mathrm{HL}$ & $10 \mathrm{~dB} \mathrm{HL}$ \\
\hline Trial 7 & Trial 8 & Trial 9 \\
\hline $15 \mathrm{~dB} H \mathrm{HL}$ & $5 \mathrm{~dB} \mathrm{HL}$ & $10 \mathrm{~dB} \mathrm{HL}$ \\
\hline Trial 10 & Trial 11 & Trial 12 \\
\hline $15 \mathrm{~dB} \mathrm{HL}$ & $20 \mathrm{~dB} \mathrm{HL}$ & $25 \mathrm{~dB} \mathrm{HL}$ \\
\hline Trial 13 & Trial 14 & \\
\hline $15 \mathrm{~dB} H L$ & $20 \mathrm{~dB}$ HL & $10 \mathrm{~dB} \mathrm{HL}$ \\
\hline
\end{tabular}

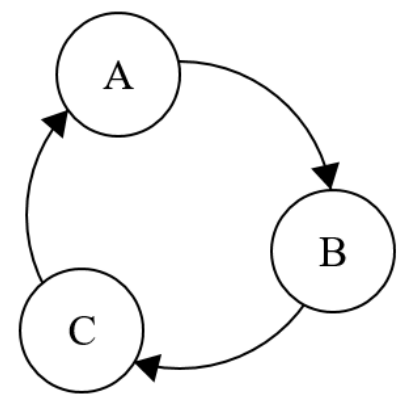

A: $\{0 \mathrm{~dB} \mathrm{HL}, 15 \mathrm{~dB} \mathrm{HL}, 30 \mathrm{~dB} H L, \ldots\}$ B: $\{-10 \mathrm{~dB} \mathrm{HL}, 5 \mathrm{~dB}$ HL, $20 \mathrm{~dB}$ HL,$\ldots\}$ C: $\{-5 \mathrm{~dB}$ HL, $10 \mathrm{~dB} \mathrm{HL}, 25 \mathrm{~dB} \mathrm{HL}, \ldots\}$

Figure 1. On the left is a typical audiometric test starting at $30 \mathrm{~dB} H L$ and ending with a threshold of 20 $d B H L$ after 14 trials. The table in middle lists the stimulus levels presented during the track in three columns. On the right is a Markov chain model of the stimulus selection procedure in which each state represents a subset of stimulus levels defined at the bottom. Obligatory transitions occur each trial in a three-step cycle through the three states.

Up-down procedures used to estimate thresholds can be modeled as a random walk in one dimension using a Markov chain (Kollmeier et al., 1988; Hoover et al., 2019). A Markov chain model of the stimulus selection procedure in Figure 1, right panel, shows that a three-step pattern can be observed whenever stimuli are selected using the down-10 dB up-5 dB rule. Each state of the Markov model represents a subset of possible stimulus levels corresponding to the columns in Figure 1, middle panel. Transitions between 
states are obligatory. After a stimulus is presented in A, the next stimulus level is always in B, and so on, until the procedure ends. The model is generic to any up-down track in which the size of the step down is twice the magnitude of a step up, regardless of step size. The following observations can be made from the Markov model:

- There are three unique sets of stimulus levels that the stimulus selection procedure cycles through each trial.

- In each set, stimulus levels are separated by the sum of a step up and a step down (i.e., $15 \mathrm{~dB}$ for down-10 dB, up-5 dB).

- A given stimulus level, and any other in the same set, can only occur every third trial.

- A stimulus level presented $t$ trials into a track will always be from the set that is $t$ mod 3 steps around the chain from the starting set, where mod is the remainder after division.

The three-step pattern is specific to the stimulus selection procedure, but the pattern should also be apparent in thresholds because the stimulus level presented on one trial is reported as threshold. The audiogram nominally reports the lowest stimulus level that will be detected in at least half of presentations. There is one stimulus level that best matches that description in any test, and it can only be presented every third trial. Thresholds obtained on other trials must be different because they are drawn from a different set. Based on the relationship between the stimulus sets shown by the Markov model, there should be a bias in thresholds obtained on different trials, and this bias should repeat every third trial. We tested this hypothesis using Monte Carlo simulations consistent with previous studies evaluating sources of procedural error in audiometric thresholds (Marshall \& Hanna, 1986; Leijon, 1992). The use of simulations facilitated the control of sources of error and the ability to generate large numbers of thresholds for the analysis of patterns of error.

\section{Method}


A simple listener model was used to generate threshold estimates in Monte Carlo simulations. The audiometric procedure was implemented according to ANSI S3.21 (ANSI, 2004), as described above. A starting level of $30 \mathrm{~dB}$ HL was used, and the procedure was stopped after the first stimulus level that met the stopping criteria (two hits and at least as many hits as misses). The listener was simulated using a cumulative Gaussian function with a slope of 0.28 calculated from the mean slope for listeners with normal hearing taken from Figure 2 of Arehart et al. (1990). Although there is no way to systematically vary the trial on which the procedure obtains a threshold without fundamentally altering the procedure, variation occurs spontaneously. A total of $10^{6}$ simulations were performed to accurately characterize this variation.

The hypothesis was that the relationships between stimulus levels shown in the Markov chain in Figure 1 would result in thresholds obtained in sequential trials that differ according to a three-step pattern. To evaluate this hypothesis, the mean and standard distribution was calculated for thresholds obtained in all simulations, as well as the mean and SD of thresholds obtained in three subsets of trials. The three subsets were defined as trials $3 k, 3 k+1$, and $3 k+2$, for integer $k$. That is, thresholds obtained on trials $7,10,13$, 16 , etc. were in one set, $8,11,14,17$, etc., were in another set, and $9,12,15,18$, etc. were in a third set. A one-way analysis of variance (ANOVA) was used to compare the means of the three sets. Simulations and analyses were performed using custom software developed for this purpose by the author.

\section{Results}

Figure 2 shows the results of $10^{6}$ threshold estimates for a simulated listener with $50 \%$ detection $\left(L_{50}\right)$ at 16.25 dB HL. The mean and SD of all thresholds were consistent with previous studies (mean $2.48 \mathrm{~dB}$ re $L_{50}$, SD 2.53 dB; Marshall \& Hanna, 1989; Leijon, 1992). When sorted by the trial on which the threshold was obtained, three different thresholds were observed $\left(3 k: \mathrm{n}=32,740\right.$, mean $=7.36 \mathrm{~dB}$ re $L_{50}, \mathrm{SD} 4.35$ $\mathrm{dB} ; 3 k+1: \mathrm{n}=279,105$, mean $=-1.25 \mathrm{~dB}$ re $L_{50}, \mathrm{SD} 0.075 \mathrm{~dB} ; 3 k+2: \mathrm{n}=689,065$ mean $=3.75 \mathrm{~dB}$ re $L_{50}$, SD $\left.0.054 \mathrm{~dB}\right)$. An ANOVA showed a significant main effect of trial subset $(\mathrm{F}(2,999,997)=4.61 \mathrm{x}$ 
$\left.10^{6}, p<0.0001, \eta_{p}^{2}=0.90\right)$. Post hoc tests with Bonferroni correction showed that the mean of each of the subsets was significantly different from the others at the $p<0.0001$ level.

The right panel of Figure 2 shows the proportion of simulations in which each stimulus level was observed as threshold. Exactly six unique stimulus levels were reported as threshold in $10^{6}$ simulations, and no stimulus level was reported in more than one subset. The three trial subsets were not reported equally often; the probability of reporting each stimulus level showed a central tendency consistent with the mean and SD of all simulations shown in the left panel.
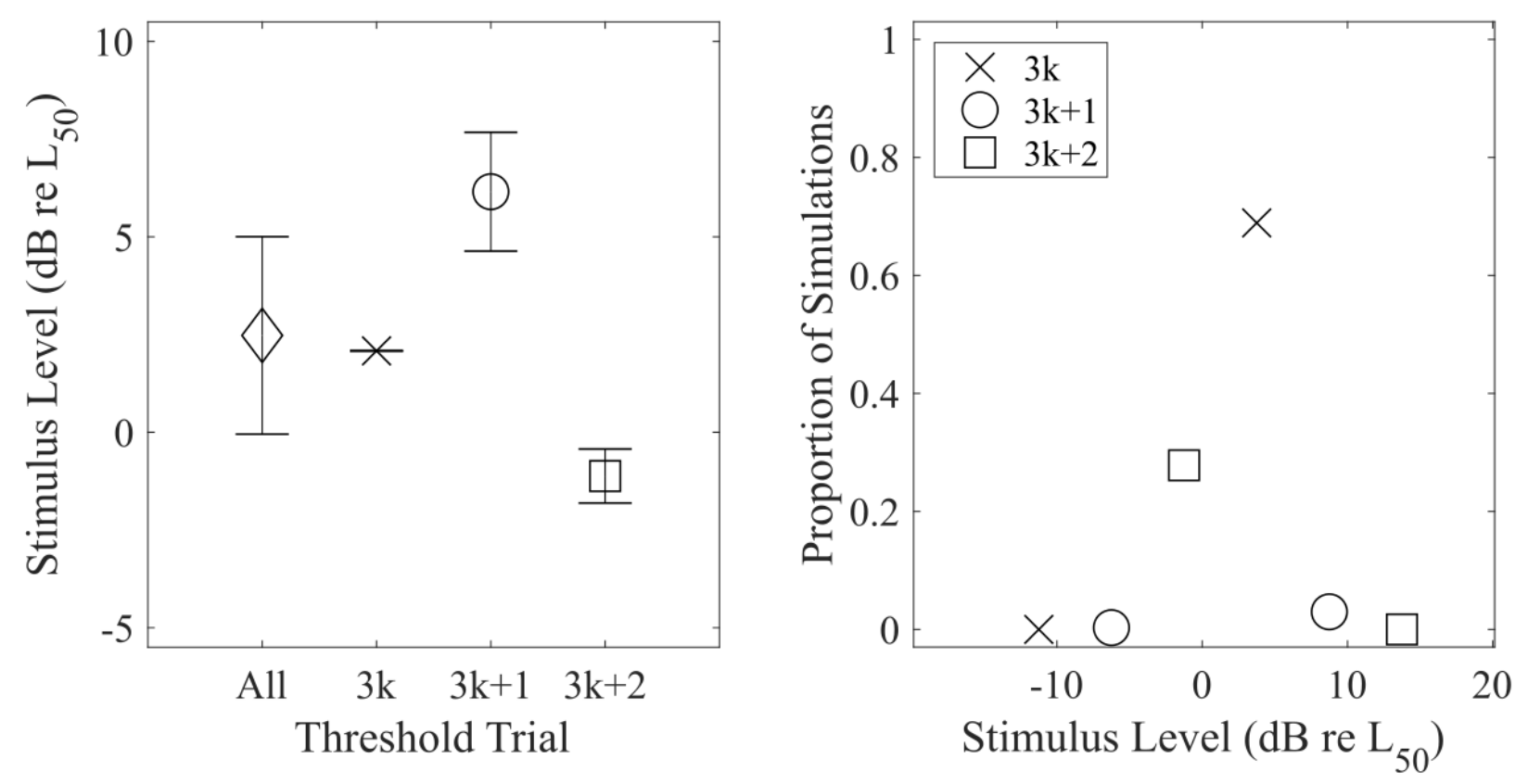

Figure 2. The mean and SD of all thresholds obtained in simulations is shown in the left panel (diamond), as well as the mean and SD of thresholds obtained on three subsets of trials. The right panel shows the proportion of simulations in which each stimulus level was reported as threshold.

\section{Discussion}

No doubt many clinicians have noticed that a single stimulus level can only be repeated every third trial in the standard audiometric procedure. However, a pattern in stimulus selection does not necessarily imply a 
pattern of error in audiometric thresholds. In the average of many tests, there is a central tendency in the stimulus levels reported as threshold independent of the three subsets of trials (Figure 2, right panel). The central tendency reflects a difference in the probability of stopping in a given subset. The subset containing the stimulus level closest to the mean of all tests was observed in a greater proportion of simulations than the other subsets. The procedure works well at the population level because it tends to stop on a trial in which the stimulus level closest to the overall mean threshold can occur. A problem arises when the result of a single test is reported on an audiogram, as is nearly always the case. One test represents one stimulus level presented during a track, which necessarily belongs to only one of the three subsets of trials. As a result, audiometric thresholds demonstrate a three-step pattern of error as a function of the trial on which threshold was obtained.

The statistics used in previous studies to describe the test-retest reliability of audiometric thresholds assume that the distribution of thresholds obtained in repeated tests in one individual (or across many individuals with the same characteristics) represents the probability distribution from which each threshold reported on an audiogram is a sample. This is only true when multiple threshold estimates are combined. Individual threshold estimates reflect the subset of the distribution containing the stimulus levels capable of being presented on a single trial. In order for each threshold reported on an audiogram to sample from the same distribution, the standard definition of threshold must be changed.

\subsection{Alternative scoring}

The three-step pattern of error can be eliminated by redefining threshold as the average of multiple trials presented during the procedure. With few trials presented, the mean of reversals typically used in laboratory psychophysics is impractical, and there is no way to guarantee equal sampling of the three Markov states with reversals. A better solution can be obtained using the Markov model of the stimulus selection procedure to select trials to average. That is, to explicitly sample the three Markov states an equal number of times. Any three sequential trials represent the three Markov states, but in staircase procedures sequential trials are highly correlated (Taylor et al., 1967). 
Equal sampling of states with minimal sequential dependence can be accomplished by selecting each stimulus level from a different loop around the Markov chain cycle. The most compact way to accomplish this is by selecting every fourth trial starting from the last known stimulus level. Counting from the stimulus level that would be presented after the final response, the score is the mean of the stimulus levels in position one, five, and nine. The shaded boxes in the middle panel of Figure 1 indicate the trials included in the average. The resulting calculation includes one stimulus level from each of the three Markov states and is always a multiple of $5 \mathrm{~dB}$ that can be plotted on an audiogram ${ }^{2}$. Thresholds obtained in this manner are systematically lower than the standard method because they represent an estimate of the distribution of all trials, whereas the standard method considers only ascending trials and is further biased upward by the stopping criteria. With a 2:1 ratio of the size of steps down and up, as in standard audiometry, the expected value of the distribution of all trials corresponds to $33.3 \%$ detection $^{3}$ (Kaernbach, 1991). Since a specific probability of detection is targeted by the procedure, the threshold obtained using the proposed method matches the definition of threshold used in all other psychophysics tasks (ANSI, 1994).

In order to evaluate the mean and SD of thresholds obtained using the proposed method, simulations were performed using the same listener model, stimulus selection procedure, and starting level as above, but a fixed number of trials were presented and threshold was calculated using the proposed method. Ten track

\footnotetext{
${ }^{2}$ This can be proved by showing that the result is always an integer multiple of 5. Sets A, B, and C are defined as above. $A=15 n$ for $n \in \mathbb{Z} . B=A+5, C=A-5$. The mean of one sample from each set is $\left(15 n_{a}+15 n_{b}+5+15 n_{c}-5\right) / 3=5 x$. This simplifies to $n_{a}+n_{b}+n_{c}=x$. Since the sum of integers is always an integer, $x \in \mathbb{Z}$. Q.E.D.

${ }^{3}$ The relationship between the magnitude of a step up and down and the probability of detection targeted by the procedure in a yes-no task is given by the equation $\Delta^{-} \varphi=\Delta^{+}(1-\varphi)$, where $\Delta^{-}$is the step down, $\Delta^{+}$is the step up, and $\varphi$ is the probability of detection targeted asymptotically (Treutwein, 1995; GarcíaPérez, 1998). For standard audiometry, $\Delta^{-}=10 \mathrm{~dB}, \Delta^{+}=5 \mathrm{~dB}$, and thus $\varphi=1 / 3$.
} 
lengths were tested, 12 to 21 trials, which excluded a minimum of four initial trials from the threshold calculation. Results of simulations are shown in Figure 3, along with simulations performed using standard audiometry with the same listener model. Unlike the standard method, a consistent distribution of thresholds was observed independent of the trial on which threshold was obtained. The mean threshold obtained using the proposed method across all simulations performed was $-1.55 \mathrm{~dB}$ re $L_{33}, \mathrm{SD} 2.91 \mathrm{~dB}$. The negative mean bias was consistent with the use of large step sizes (García-Pérez, 1998). The SD of thresholds was higher for the proposed method $(2.91 \mathrm{~dB})$ compared to the standard method $(2.53 \mathrm{~dB})$. This can be observed when looking at the proportion of simulations in which each stimulus level was reported as threshold by both procedures in Figure 3, right panel. However, the $2.53 \mathrm{~dB}$ SD of the standard procedure can only be obtained by averaging across multiple threshold estimates because of the three-step pattern reported here. Each threshold obtained with the standard method is a sample of one of the three subsets of trials, which have three different means (as shown in Figure 2, left panel). Using the proposed scoring method, each threshold is a random sample of the distribution of all trials, accurately described by a SD of $2.91 \mathrm{~dB}$.
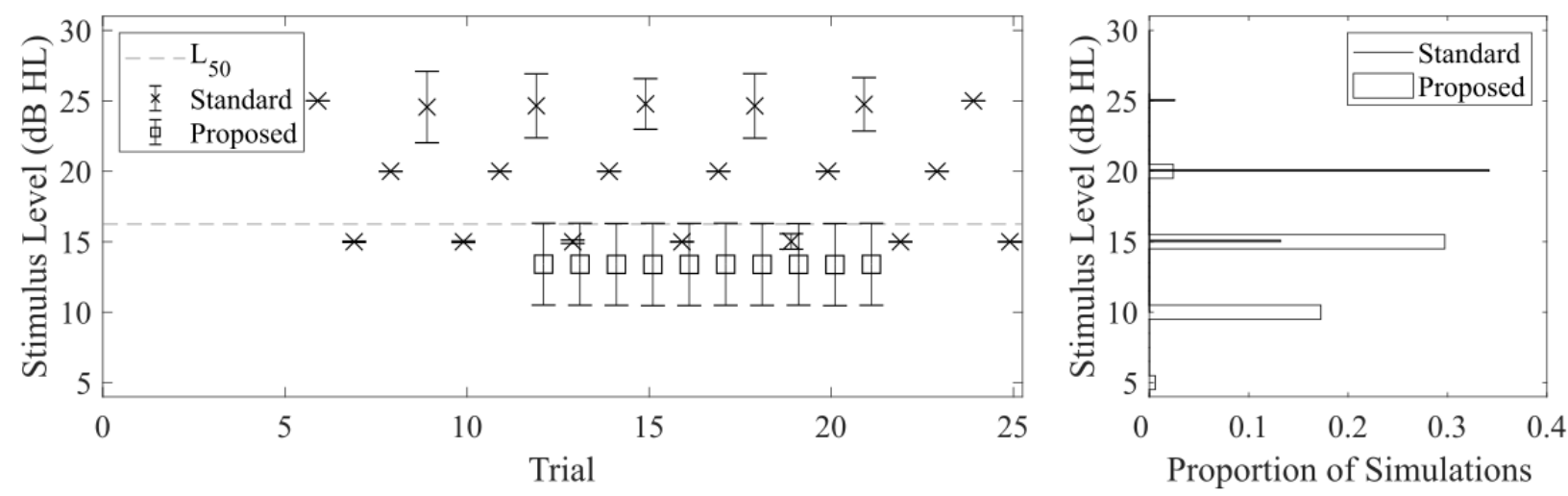

Figure 3. The left panel shows the mean and SD thresholds obtained in $10^{6}$ Monte Carlo simulations as a function of the trial on which threshold was obtained. The stimulus level at 50\% detection, $L_{50}$, is shown (dashes). Thresholds obtained using the standard method ( $x$ ) show a three-step pattern as a function of trial. For the proposed scoring method (square), $10^{5}$ tracks were simulated in each of 10 fixed-length 
tracks ranging from 12 to 21 trials. No change in threshold as a function of trial was observed with the proposed method. The right panel shows the proportion of simulations in which each stimulus level was reported as threshold.

\section{Conclusions}

Each threshold reported on the audiogram is typically the result of a single test. A systematic bias in thresholds that would otherwise "average out" due to central tendency in the probability of reporting threshold from a given trial group, instead persists. A Markov chain model of the stimulus selection procedure showed a three-step pattern in the stimulus levels that can be presented each trial. When trials were sorted into three groups, mean thresholds obtained on trials in each group reflected a bias consistent with the Markov model. Thus, a three-step pattern was described in the error of audiometric thresholds.

An alternative scoring method is proposed that equally samples each of the Markov states. The alternative threshold has a greater SD than the standard method, but, unlike the standard method, each threshold estimate is a random sample of the same distribution rather than one of three parts of a distribution dependent on the trial on which it was obtained. The resulting threshold represents a specific probability of detection and may be preferable for some clinical and research applications.

Reducing the error of audiometric threshold estimates can benefit the diagnosis of hearing loss, the detection of a change in hearing, and improve our ability to quantify and control audibility. The error described here represents a pattern in the stimulus selection procedure that persists in thresholds reported on the audiogram because of the lack of averaging at any step of the process. The proposed method addresses this by introducing averaging into the calculation of each threshold. Future work will further evaluate the consequences of the three-step pattern for the detection of a change in hearing and the potential benefit of using the proposed scoring method in the measurement and control of audibility.

\section{Acknowledgements}


This work was supported by the National Institutes of Health National Institute for Deafness and Other Communication Disorders (R01 DC015051 to Frederick J. Gallun), and by startup funding from the University of Maryland. A special thank you to Michelle Molis, Frederick Gallun, and Katherine Palandrani for providing critical feedback in the development of this manuscript.

\section{References}

ANSI/ASA. (2009). S3.21-2009 Methods for Manual Pure-Tone Threshold Audiometry. New York, NY, US: American National Standards Institute.

ANSI/ASA. (1994). S1.1-1994 Acoustical Terminology. New York, NY, US: American National Standards Institute.

Arehart, K. H., Burns, E. M., \& Schlauch, R. S. (1990). “A comparison of psychometric functions for detection in normal-hearing and hearing-impaired listeners.” J. Speech Lang. Hear. Res. 33 (3), $433-439$.

Bunch, C. C. (1943). Clinical audiometry. St. Louis, MO, US: Mosby.

Carhart, R., \& Jerger, J. F. (1959). "Preferred method for clinical determination of pure-tone thresholds." J. Speech Hear. Dis. 24(4), 330-345.

Flamme, G. A., Geda, K., McGregor, K. D., Wyllys, K., Deiters, K. K., Murphy, W. J., \& Stephenson, M. R. (2015). “Stimulus and transducer effects on threshold.” Int. J. Audiol. 54(sup1), S19-S29.

García-Pérez, M. A. (1998). "Forced-choice staircases with fixed step sizes: asymptotic and small-sample properties.”Vision Res. 38 (12), 1861-1881.

Green, D. M., Kidd Jr, G., \& Stevens, K. N. (1987). "High-frequency audiometric assessment of a young adult population.” J. Acoust. Soc. Am. 81(2), 485-494. 
Harris, J. D. (1979). “Optimum threshold crossings and time-window validation in threshold pure-tone computerized audiometry.” J. Acoust. Soc. Am. 66(5), 1545-1547.

Hirsh, I. J. (1952). The measurement of hearing. New York, NY, US: McGraw-Hill.

Hoover, E. C., Gallun, F. J., \& Eddins, D. A. (2019). "Challenging standard practices in adaptive psychophysics.” J. Acoust. Soc. Am. 145(3), 1758-1758.

ISO. (2010). ISO 8253-1 Acoustics - Audiometric test methods - Part 1: Pure-tone air and bone conduction audiometry. Geneva, Switzerland: International Organization for Standardization.

Jerlvall, L., \& Arlinger, S. (1986). "A comparison of 2-dB and 5-dB step size in pure-tone audiometry.” Scand Audiol, 15(1), 51-56.

Kaernbach, C., et al. (1991). "Simple adaptive testing with the weighted up-down method." Percept. Psychophys. 49 (3), 227-229.

Kollmeier, B., Gilkey, R. H., \& Sieben, U. K. (1988). “Adaptive staircase techniques in psychoacoustics: A comparison of human data and a mathematical model.” J. Acoust. Soc. Am. 83(5), 1852-1862.

Lecluyse, W., \& Meddis, R. (2009). “A simple single-interval adaptive procedure for estimating thresholds in normal and impaired listeners." J. Acoust. Soc. Am. 126(5), 2570-2579.

Leijon, A. (1992). “Quantization error in clinical pure-tone audiometry.” Scand. Audiol. 21(2), 103-108.

Mahomed, F., Swanepoel, D. W., Eikelboom, R. H., \& Soer, M. (2013). "Validity of automated threshold audiometry: a systematic review and meta-analysis.” Ear Hear. 34 (6), 745-752.

Margolis, R. H., Wilson, R. H., Popelka, G. R., Eikelboom, R. H., Swanepoel, D. W., \& Saly, G. L. (2015). “Distribution characteristics of normal pure-tone thresholds.” Int. J. Audiol. 54 (11), 796-805. 
Marshall, L., \& Hanna, T. E. (1986). Stopping rules for audiological ascending test procedures: Computer simulation evaluation (NSMRL Report No. 1081). Groton, CT, US: Naval Submarine Medical Research Lab.

Marshall, L., \& Hanna, T. E. (1989). "Evaluation of stopping rules for audiological ascending test procedures using computer simulations.” J. Speech Lang. Hear. Res. 32 (2), 265-273.

Marshall, L., Hanna, T. E., \& Wilson, R. H. (1996). "Effect of step size on clinical and adaptive 2IFC procedures in quiet and in a noise background.” J. Speech Lang. Hear. Res. 39 (4), 687-696.

Marshall, L., \& Jesteadt, W. (1986). "Comparison of pure-tone audibility thresholds obtained with audiological and two-interval forced-choice procedures.” J. Speech Lang. Hear. Res. 29 (1), 8291.

Meddis, R., \& Lecluyse, W. (2011). "The psychophysics of absolute threshold and signal duration: a probabilistic approach.” J. Acoust. Soc. Am. 129 (5), 3153-3165.

Stelmachowicz, P. G., Beauchaine, K. A., Kalberer, A., Kelly, W. J., \& Jesteadt, W. (1989). "Highfrequency audiometry: Test reliability and procedural considerations.” J. Acoust. Soc. Am. 85 (2), 879-887.

Stelmachowicz, P. G., Beauchaine, K. A., Kalberer, A., Langer, T., \& Jesteadt, W. (1988). "The reliability of auditory thresholds in the 8-to $20-\mathrm{kHz}$ range using a prototype audiometer.” J. Acoust. Soc. Am. $83(4), 1528-1535$.

Souza, N. N., Dhar, S., Neely, S. T., \& Siegel, J. H. (2014). “Comparison of nine methods to estimate earcanal stimulus levels.” J. Acoust. Soc. Am. 136(4), 1768-1787.

Taylor, M. M., Forbes, S. M., \& Creelman, C. D. (1967). "Sequential Dependence in Signal Detection, and Parameter Estimation by Sequential Tracking (PEST).” J. Acoust. Soc. Am. 42(5), 1195-1195.

Treutwein, B. (1995). “Adaptive psychophysical procedures.” Vision Res. 35 (17), 2503-2522. 